\title{
Diagnostic Value of the Methylation of Multiple Gene Promoters in Serum in Hepatitis B Virus-Related Hepatocellular Carcinoma
}

\author{
Xueyan Dong, ${ }^{1}$ Qiang Hou, ${ }^{2}$ Yueming Chen, ${ }^{1}$ and Xianjun Wang ${ }^{1}$ \\ ${ }^{1}$ Department of Laboratory Medicine, Hangzhou First People's Hospital, Hangzhou, Zhejiang 310006, China \\ ${ }^{2}$ Hangzhou Cancer Institution, Hangzhou Cancer Hospital, Hangzhou, Zhejiang 310002, China \\ Correspondence should be addressed to Yueming Chen; cymwly1972@163.com
}

Received 15 March 2017; Accepted 4 July 2017; Published 29 August 2017

Academic Editor: Paola Gazzaniga

Copyright ( 2017 Xueyan Dong et al. This is an open access article distributed under the Creative Commons Attribution License, which permits unrestricted use, distribution, and reproduction in any medium, provided the original work is properly cited.

\begin{abstract}
This study sought to evaluate the diagnostic value of the methylation of multiple gene promoters in serum in hepatitis $\mathrm{B}$ virus- (HBV-) related hepatocellular carcinoma (HCC). A total of 343 participants were enrolled, including 98 patients with HCC, 75 patients with liver cirrhosis (LC), 90 patients with chronic hepatitis B (CHB), and 80 healthy individuals. RASSF1A, APC, BVES, TIMP3, GSTP1, and HOXA9 were selected as the candidate genes. The MethyLight method was used to assay promoter methylation statuses. The diagnostic performances of markers were assessed by constructing receiver operating characteristic (ROC) curves. The prevalences of methylation for RASSF1A, APC, BVES, HOXA9, GSTP1, and TIMP3 were $52.04 \%, 36.73 \%, 29.59 \%, 20.41 \%, 17.35 \%$, and $11.22 \%$, respectively. APC methylation completely overlapped with RASSF1A methylation. The area under the curve (AUC) for RASSF1A methylation (0.718) was better than the corresponding AUC for AFP (0.609) in distinguishing HCC from CHB. When RASSF1A, BVES, HOXA9, and AFP were combined, the AUC was 0.852 (95\% CI $=0.796-0.908, P=0.028$ ), and the sensitivity and specificity were $83.7 \%$ and $78.9 \%$, respectively. In conclusion, an assay that combines methylation of the RASSF1A, BVES, and HOXA9 gene promoters in serum and AFP could significantly improve HBV-related HCC diagnoses.
\end{abstract}

\section{Introduction}

Hepatocellular carcinoma (HCC) is one of the most common human malignant tumors and causes an estimated 50 million deaths per year worldwide $[1,2]$. Chronic hepatitis $B$ virus (HBV) infection is a major cause of HCC in East Asia. Currently, a serum alpha fetoprotein (AFP) assay is commonly used to detect HCC. However, this assay has relatively low sensitivity and specificity; therefore, its clinical application is limited. Given current clinical diagnostic tools, patients with HCC may not undergo effective treatment. Thus, most such patients have already progressed to an advanced disease stage by the time they are definitively diagnosed. Therefore, there is an urgent need to identify effective biomarkers for HCC.

$\mathrm{CpG}$ island methylation in the gene promoter is an important epigenetic mechanism that is often involved in carcinogenesis. Certain tumor suppressor genes and other pivotal genes that regulate cell signaling pathways are frequently silenced in tumor tissues due to promoter hypermethylation [3-5]. These epigenetic changes have been tested and shown to be potential markers for HCC [6]. However, it is inconvenient and invasive to use tumor tissues to detect promoter methylation in high-risk populations. Serum DNA from patients with cancer is derived from apoptotic cells, necrotic cells, or circulating tumor cells and reflects a variety of DNA changes in the forms of allelic imbalance, DNA integration, mutation, and methylation [7-9]. These changes in serum DNA are highly consistent with those present in tumor tissues $[10,11]$. Prior literature indicates that the methylation of gene promoters in serum could be a promising noninvasive biomarker for diagnosing HCC.

In this study, RAS association domain family protein $1 \mathrm{~A}$ (RASSF1A), adenomatous polyposis coli (APC), blood vessel 
epicardial substance ( $B V E S)$, TIMP metallopeptidase inhibitor 3 (TIMP3), glutathione S-transferase pi 1 (GSTP1), and homeobox A9 (HOXA9) were selected as candidate targets; these genes are frequently methylated during carcinogenesis of digestive tract carcinoma [12-15]. The MethyLight method [16] was used to measure serum methylation statuses of genes in patients with HCC, patients with liver cirrhosis (LC), patients with chronic hepatitis $\mathrm{B}(\mathrm{CHB})$, and healthy subjects. Subsequently, the diagnostic performances of the selected markers were assessed by constructing receiver operating characteristic (ROC) curves.

\section{Materials and Methods}

2.1. Study Population. A total of 343 participants who visited Hangzhou First People's Hospital from January 2011 to December 2015 were enrolled in the study. They were divided into four age- and gender-matched groups (HCC patients, LC patients, CHB patients, and healthy subjects). In detail, the 98 patients with HCC had been diagnosed via liver ultrasound, computed tomography (CT), serum AFP level, and ultimately histological examination. The 75 patients with LC had been diagnosed via liver ultrasound and CT and exhibited LC accompanied by portal hypertension and hypersplenism. The 90 patients with $\mathrm{CHB}$ satisfied diagnostic criteria based on guidelines for the prevention and treatment of chronic hepatitis B (2010 version) issued by the Chinese Society of Hepatology and the Chinese Society of Infectious Diseases of the Chinese Medical Association. Additionally, the HCC patients, LC patients, and CHB patients had HBV surface antigen- (HBsAg-) positive serum. Subjects who presented with other liver diseases, such as autoimmune hepatitis, alcoholic hepatitis, and infection with another type of hepatitis virus, were excluded from the study. Eighty healthy individuals were obtained from the Physical Examination Center of Hangzhou First People's Hospital. All subjects provided written informed consent, and this study was approved by the ethics committee of Hangzhou First People's Hospital.

2.2. Serum DNA Extraction and Sodium Bisulfite Treatment. Five-milliliter samples of peripheral blood were drawn from the patients and healthy subjects. Samples were centrifuged at $2000 \times \mathrm{g}$ for $10 \mathrm{~min}$. Subsequently, $2 \mathrm{ml}$ of serum was collected from each sample via centrifugation at $12000 \times \mathrm{g}$ for $5 \mathrm{~min}$ and stored at $-80^{\circ} \mathrm{C}$ until use. A serum DNA extraction kit (GenMagBio Biotechnology Co. Ltd., Beijing, China) was used to extract DNA from $600 \mu \mathrm{l}$ of serum. Serum DNA was modified via sodium bisulfite treatment and purified using the EpiTect Bisulfite Kit (Qiagen, Hilden, Germany). The aforementioned operations were performed in accordance with the protocols recommended by the manufacturer.

2.3. Preparation of Positive Control. One reaction was performed for in vitro methylation. The $20 \mu \mathrm{l}$ reaction system consisted of $2 \mu \mathrm{l}$ of $10 \times$ NEBuffer, $1 \mu \mathrm{l}$ of genomic DNA $(15 \mu \mathrm{g} / \mathrm{l})$ from umbilical cord blood from a healthy fetus, $2 \mu \mathrm{l}$ of S-adenosylmethionine (SAM) $(1600 \mu \mathrm{M}), 1 \mu \mathrm{l}$ of

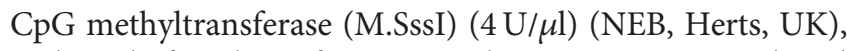
and $14 \mu \mathrm{l}$ of nuclease-free water. This mixture was incubated at $37^{\circ} \mathrm{C}$ for $1 \mathrm{~h}$, and the reaction was then stopped at $65^{\circ} \mathrm{C}$ for $20 \mathrm{~min}$. Methylated genomic DNA was treated and purified using the sodium bisulfite modification approach described above.

2.4. DNA Methylation Assay. The methylation status of each gene was examined using methylation-specific quantitative PCR (MethyLight). The sequences of the primers and probes for MethyLight were previously described [17-22]; these primers and probes were synthesized by Shanghai HuiRui Biotechnology Co. Ltd. (Table 1). In this study, actin beta $(A C T B)$ was used as the internal reference gene to correct for differences in DNA template quantities among samples. The PCR mixture had a final volume of $20 \mu \mathrm{l}$ and contained $1 \mu \mathrm{l}$ of bisulfite-treated DNA, $0.15 \mu \mathrm{l}$ of each primer $(10 \mu \mathrm{M}), 0.1 \mu \mathrm{l}$ of each probe $(10 \mu \mathrm{M}), 9.6 \mu \mathrm{l}$ of nucleasefree water, and $10.0 \mu \mathrm{l}$ of $2 \times$ PCR Buffer (Toyobo Co. Ltd., Japan), which consisted of Taq DNA polymerase, reaction buffer, and a deoxynucleotide triphosphate mixture. PCR was performed using an ABI 7500 Sequence Detection System (Life Technologies, USA). The PCR program included an initial denaturation step at $95^{\circ} \mathrm{C}$ for $3 \mathrm{~min}$ followed by 45 cycles of denaturation at $95^{\circ} \mathrm{C}$ for $10 \mathrm{~s}$ and annealing at $60^{\circ} \mathrm{C}$ for $1 \mathrm{~min}$. M.SssI-treated DNA, normal lymphocyte DNA, and nuclease-free water were used as a positive control, a negative control, and a blank control, respectively. Each sample was assessed in duplicate, with the average of the two duplicates used for analysis. Gene promoter methylation statuses are presented as percentage of methylated reference (PMR) values [23]. A PMR $\geq 4 \%$ was classified as positive, whereas a $\mathrm{PMR}<4 \%$ was classified as negative; this threshold has been validated in the literature as the standard cut-off value for PMR [24-26].

2.5. Statistical Analysis. The Mann-Whitney $U$ test was used to examine differences in nonparametric variables. Associations between methylation and clinicopathologic parameters were determined using chi-square $\left(\chi^{2}\right)$ tests. Diagnostic efficacies were presented as areas under ROC curves (AUCs). $P$ values $<0.05$ were regarded as statistically significant. All data analyses were performed using SPSS software, version 21 (IBM, Armonk, NY, USA).

\section{Results}

3.1. Demographic Characteristics. General clinical information was collected for 343 subjects. Serum levels of alanine aminotransferase (ALT), albumin (ALB), total bilirubin (TBIL), AFP, and blood platelet (PLT) count significantly differed among the four groups (all $P<0.05$ ). In particular, relative to the healthy control group, the HCC, LC, and CHB groups had higher ALT, TBIL, and AFP levels but lower ALB and PLT levels (all $P<0.05$ ).

3.2. Serum Methylation Statuses of Multiple Gene Promoters. Rates of hypermethylation of RASSF1A, APC, BVES, TIMP3, GSTP1, and HOXA9 promoters in HCC patients, LC patients, $\mathrm{CHB}$ patients, and healthy individuals are shown 
TABLE 1: List of primers and probes for MethyLight.

\begin{tabular}{|c|c|}
\hline Gene & Primer and TaqMan probe sequences $\left(5^{\prime} \rightarrow 3^{\prime}\right)$ \\
\hline \multirow{3}{*}{ RASSF1A } & Forward primer: GCGTTGAAGTCGGGGTTC \\
\hline & Reverse primer: CCCGTACTTCGCTAACTTTAAACG \\
\hline & TaqMan probe: FAM-FAM-ACAAACGCGAACCGAACGAAACCA-BHQ1 \\
\hline \multirow{3}{*}{$A P C$} & Forward primer: AGTGCGGGTCGGGAAGC \\
\hline & Reverse primer: AACCACATATCGATCACGTACG \\
\hline & TaqMan probe: FAM-AAAACGCCCTAATCCGCATCCAACG-BHQ1 \\
\hline \multirow{3}{*}{$B V E S$} & Forward primer: GGACGGAGTGGGCGATATC \\
\hline & Reverse primer: CCTCGAACCGCGCAAA \\
\hline & TaqMan probe: FAM-CCTACGTACAACCGAACG-MGB \\
\hline \multirow{3}{*}{ TIMP3 } & Forward primer: GCGTCGGAGGTTAAGGTTGTT \\
\hline & Reverse primer: CTCTCCAAAATTACCGTACGCG \\
\hline & TaqMan probe: FAM-AACTCGCTCGCCCGCCGAA-BHQ1 \\
\hline \multirow{3}{*}{ GSTP1 } & Forward primer: CGTCGTGATTTAGTATTGGGGC \\
\hline & Reverse primer: CTAATAACGAAAACTACGACGACGAAA \\
\hline & TaqMan probe: FAM-ATAAGGTTCGGAGGTCGCGAGGTTTTCGT-BHQ1 \\
\hline \multirow{3}{*}{ HOXA9 } & Forward primer: AATAAATTTTATCGTAGAGCGGTAC \\
\hline & Reverse primer: CATATAACAACTTAATAACACCGAA \\
\hline & TaqMan probe: FAM-GCGCCCCCATTAACCGTACGCGT-BHQ1 \\
\hline \multirow{3}{*}{$A C T B$} & Forward primer: TGGTGATGGAGGAGGTTTAGTAAGT \\
\hline & Reverse primer: AACCAATAAAАCСТАСТССТСССТТАA \\
\hline & TaqMan probe: FAM-ACCACCACCCAACACACAATAACAAACACA-BHQ1 \\
\hline
\end{tabular}

TABLE 2: The positive rates of methylation for promoters of 6 genes in serum $[n(\%)]$.

\begin{tabular}{|c|c|c|c|c|c|c|c|c|}
\hline Gene & HCC patients $(n=98)$ & LC patients $(n=75)$ & CHB patients $(n=90)$ & Healthy controls $(n=80)$ & $\chi^{2 \mathrm{a}}$ & $P^{\mathrm{a}}$ & $\chi^{2 \mathrm{~b}}$ & $P^{\mathrm{b}}$ \\
\hline RASSF1A & $51(52.04)$ & $10(13.33)$ & $4(4.44)$ & $3(3.75)$ & 26.215 & 0.000 & 49.078 & 0.000 \\
\hline$A P C$ & $36(36.73)$ & $4(5.33)$ & $2(2.22)$ & $0(0)$ & 21.834 & 0.000 & 32.543 & 0.000 \\
\hline BVES & $29(29.59)$ & $3(4.00)$ & $1(1.11)$ & $0(0)$ & 16.799 & 0.000 & 26.292 & 0.000 \\
\hline TIMP3 & $11(11.22)$ & $2(2.67)$ & $0(0)$ & $0(0)$ & 4.477 & 0.034 & 10.730 & 0.001 \\
\hline GSTP1 & $17(17.35)$ & $2(2.67)$ & $0(0)$ & $0(0)$ & 9.365 & 0.002 & 17.164 & 0.000 \\
\hline HOXA9 & $20(20.41)$ & $4(5.33)$ & $3(3.33)$ & $0(0)$ & 8.081 & 0.004 & 10.863 & 0.001 \\
\hline
\end{tabular}

HCC: hepatocellular carcinoma; LC: liver cirrhosis; CHB: chronic hepatitis B. ${ }^{a} \mathrm{HCC}$ patients versus LC patients, $P<0.05$. ${ }^{\mathrm{b}} \mathrm{HCC}$ patients versus CHB patients, $P<0.01$.

in Table 2. In HCC patients, the prevalences of hypermethylation for RASSF1A, APC, BVES, HOXA9, GSTP1, and TIMP3 were 52.04\%, 36.73\%, 29.59\%, 20.41\%, 17.35\%, and $11.22 \%$, respectively, and $A P C$ methylation completely overlapped with RASSF1A methylation. In addition, RASSF1A methylation was sometimes detected in serum from LC patients $(13.33 \%)$ but infrequently observed in serum from healthy subjects (3.75\%). The other 5 genes showed low methylation rates in LC patients $(2.67 \%-5.33 \%)$ and no detected methylation in healthy subjects.

3.3. Powers of Methylation Statuses of Multiple Genes and the AFP Assay to Distinguish HCC from $C H B$. For discriminating between HCC and CHB, the sensitivity of RASSF1A methylation in serum was greater than the sensitivities of the other diagnostic indicators, whereas the sensitivities of $B V E S$, APC, TIMP3, GSTP1, and HOXA9 methylation in serum were all lower than the sensitivity of AFP $(\geq 20 \mathrm{ng} / \mathrm{l})$
TABle 3: Powers of the methylation statuses of multiple genes and the AFP assay for distinguishing HCC from $\mathrm{CHB}$.

\begin{tabular}{lccc}
\hline Indicator & Sensitivity (\%) & Specificity (\%) & AUC \\
\hline RASSF1A & 52.0 & 91.5 & 0.718 \\
APC & 36.7 & 96.4 & 0.650 \\
BVES & 29.6 & 97.6 & 0.636 \\
TIMP3 & 11.2 & 98.8 & 0.356 \\
GSTP1 & 17.4 & 98.7 & 0.486 \\
HOXA9 & 20.4 & 95.8 & 0.521 \\
AFP $(\geq 20 \mathrm{ng} / \mathrm{l})$ & 48.0 & 73.9 & 0.609 \\
\hline
\end{tabular}

HCC: hepatocellular carcinoma; CHB: chronic hepatitis B; AUC: area under the curve.

(Table 3). For all 6 genes, the specificities of promoter methylation in serum were better than the specificity of AFP $(\geq 20 \mathrm{ng} / \mathrm{l})$. Because APC methylation completely overlapped 


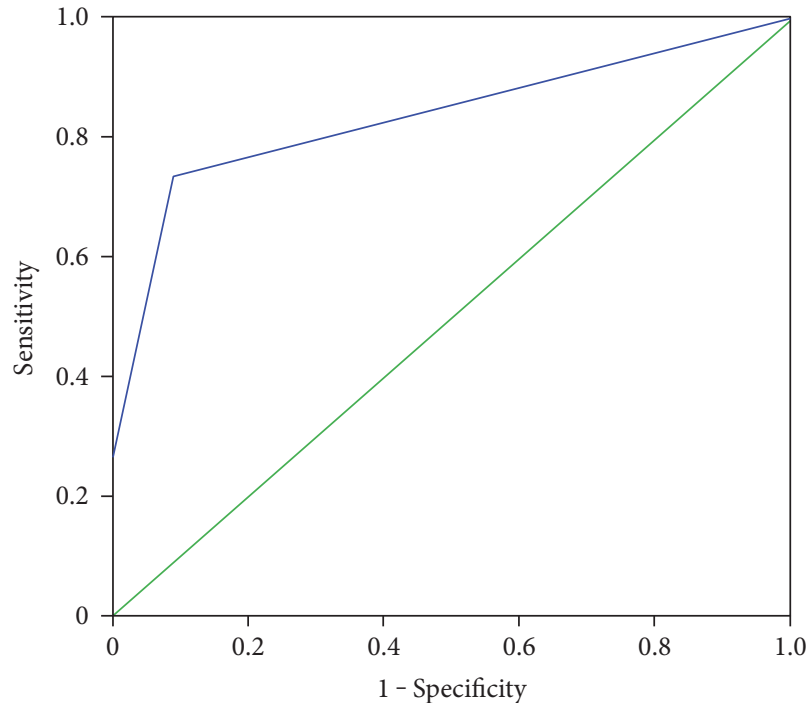

(a)

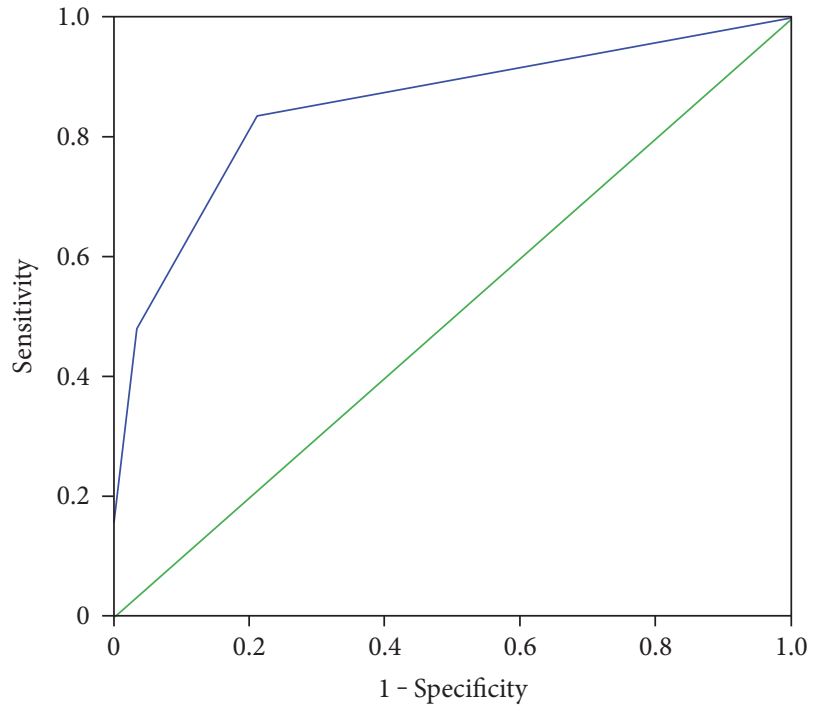

(b)

FIgURE 1: Receiver operating characteristic (ROC) curves analyzing the use of RASSF1A, BVES, and HOXA9 methylation in serum and AFP $(\geq 20 \mathrm{ng} / \mathrm{l})$ for distinguishing hepatocellular carcinoma (HCC) from chronic hepatitis B (CHB). (a) When RASSF1A, BVES, and HOXA9 methylation statuses in serum were utilized together, the AUC was $0.834(95 \% \mathrm{CI}=0.774-0.894, P=0.031)$, the sensitivity was $73.5 \%$, and the specificity was $91.1 \%$. (b) When RASSF1A, BVES, and HOXA9 methylation statuses in serum and AFP were combined, the AUC was $0.852(95 \% \mathrm{CI}=0.796-0.908, P=0.028)$ and the sensitivity and specificity were $83.7 \%$ and $78.9 \%$, respectively.

with RASSF1A methylation, the AUCs of serum RASSF1A methylation (0.718), BVES methylation (0.636), AFP $(\geq 20 \mathrm{ng} / \mathrm{l})$ (0.609), and HOXA9 methylation (0.521) indicated that these metrics were the top 4 indicators for distinguishing between HCC and CHB.

3.4. Powers of Combined Assays to Distinguish HCC from $C H B$. To further investigate the diagnostic value of combining methylation statuses of RASSF1A, BVES, and HOXA9 in serum and AFP $(\geq 20 \mathrm{ng} / \mathrm{l})$ to distinguish HCC from $\mathrm{CHB}, \mathrm{ROC}$ curves were constructed. The results showed that when methylation statuses of RASSF1A, BVES, and HOXA9 in serum were utilized together, the AUC was 0.834 (95\% $\mathrm{CI}=0.774-0.894, P=0.031)$ and the sensitivity and specificity were $73.5 \%$ and $91.1 \%$, respectively. For a combination of the methylation statuses of RASSF1A, BVES, and HOXA9 in serum and AFP $(\geq 20 \mathrm{ng} / \mathrm{l})$, the AUC was 0.852 (95\% CI $=0.796-0.908, P=0.028)$ and the sensitivity and specificity were $83.7 \%$ and $78.9 \%$, respectively (Figure 1).

\section{Discussion}

In areas where $\mathrm{HBV}$ infection is highly prevalent, a chronic liver disease spectrum has been formed that extends from CHB to LC and even to progression to HCC $[1,2]$. Therefore, having patients with chronic HBV infection undergo regular assessments using a powerful indicator would help improve HCC diagnoses and the timeliness of treatment. Aberrant gene promoter hypermethylation has been proposed as an approach for diagnosing solid tumors. Specifically, an assay of DNA methylation in serum could be a noninvasive method for assessing a reliable biomarker for tumors.
In this study, a magnetic bead method was used to isolate and purify serum DNA, and the MethyLight method was used to perform the DNA methylation assay. These methods guaranteed the reliability of the study results. Six tumorassociated genes (RASSF1A, APC, BVES, TIMP3, GSTP1, and HOXA9) were selected as candidates. These genes are involved in a variety of cellular functions and signaling pathways, such as cell proliferation, invasion and adhesion (RASSF1A, APC, and BVES), metastasis and angiogenesis (TIMP3), detoxification (GSTP1), and cell differentiation (HOXA9) [12-15]. We found that the highest rate of methylation was observed for RASSF1A (52.04\%, 51/98), followed by APC $(36.73 \%, 36 / 98)$, and that APC methylation completely overlapped with RASSF1A methylation. Notably, methylated RASSF1A and methylated APC were both sometimes detected in $\mathrm{LC}$ and $\mathrm{CHB}$. These results imply that methylation of RASSF1A and APC may be a common aberrant epigenetic change during the development of HCC and could even be involved in early stages of hepatocarcinogenesis.

Comparisons indicated that RASSF1A methylation, BVES methylation, AFP $(\geq 20 \mathrm{ng} / \mathrm{l})$ and HOXA9 methylation were the top 4 biomarkers for distinguishing HCC from CHB but that only RASSF $1 A$ methylation exhibited better sensitivity (52.0\%) and specificity (91.5\%) than AFP $(48.0 \%$ and $73.9 \%$, resp.). Cell-free methylated RASSF1A exhibited large discrepancies with respect to diagnostic performance, including wide ranges for sensitivity (0.27 to 0.94$)$ and specificity (0.38 to 0.95$)$; this phenomenon was likely due to HCC heterogeneity and the selected methylation assay method [27]. Furthermore, we tested the efficacy of combined assays. A combined assay that included the methylation statuses of RASSF1A, BVES, 
and HOXA9 in serum and AFP $(\geq 20 \mathrm{ng} / \mathrm{l})$ exhibited an improved AUC (0.852), sensitivity (83.7\%), and specificity (78.9\%). Recently, Lu et al. [28] screened 4 hypermethylated genes (APC, COX2, RASSF1A, and miR-203) for diagnosing $\mathrm{HBV}$-related $\mathrm{HCC}$ using a high-throughput approach. In that study, the 4 biomarkers were combined to form a plasma methylation predictive panel that achieved a sensitivity of $84.2 \%$, a specificity of $83.0 \%$, and an AUC of 0.87 with respect to discriminating between HBV-related HCC and noncancerous control samples. These data illuminated the high diagnostic potential of methylated markers in cell-free DNA from HCC patients. In addition, several literature reports have demonstrated correlations between clinicopathological characteristics of HCC, including clinical prognosis, and such markers [27-29].

Currently, molecular pathologic epidemiology (MPE) is an emerging field of epidemiology based on molecular classification of cancer [30]. MPE research links between various exposures and molecular pathology. Similarly, it can be expanded with circulating biomarkers. The involvement of HBV infection in epigenetic alternations during hepatocarcinogenesis has been described; HBV X ( $\mathrm{HBx})$ protein expression promoted DNA methyltransferase (DNMT) activity by upregulation of DNMT1, DNMT3A1, and DNMT3A2 and selectively facilitated regional hypermethylation of specific tumor suppressor genes [4]. In combination of the results of this study, circulating methylated biomarkers are worthy to identify in HBV-related HCC in the future. On the other hand, one case-control study suggested that a disintegrin and metalloproteinase with thrombospondin motifs 5 (ADAMTS5) polymorphism was identified to be a useful marker for aflatoxin B1- (AFB1-) related HCC diagnosis and prognosis [31]. Hence, integrative analysis of various exposures and molecular markers is the fundamental premise of precision medicine for HCC. Fortunately, it is increasingly feasible to apply advanced omics technologies to screen specific cancer datasets; this advancement has provided enormous opportunities for molecular classification, personalized prevention, and therapy for the highly heterogeneous diseases including HCC [32]. Nevertheless, there are some challenges in MPE research especially with respect to selection bias, sample size limitations, measurement error and multidisciplinary research environment, and so forth [30].

\section{Conclusions}

In summary, an assay that combines methylation of the RASSF1A, BVES, and HOXA9 gene promoters in serum and AFP could significantly improve HCC diagnoses for patients with chronic HBV infection.

\section{Conflicts of Interest}

The authors declare that no financial competing interests exist.

\section{Acknowledgments}

This work was supported by the Hangzhou Scientific and Technological Commission (20140733Q14), Zhejiang Province, China.

\section{Referencess}

[1] S. K. Fung and A. S. Lok, "Management of patients with hepatitis B virus-induced cirrhosis," Journal of Hepatology, vol. 42, no. 1, pp. S54-S64, 2005.

[2] M. Tanaka, F. Katayama, H. Kato et al., "Hepatitis B and C virus infection and hepatocellular carcinoma in China: a review of epidemiology and control measures," Journal of Epidemiology, vol. 21, no. 6, pp. 401-416, 2011.

[3] I. Tischoff and A. Tannapfel, "DNA methylation in hepatocellular carcinoma," World Journal of Gastroenterology, vol. 14, no. 11, pp. 1741-1748, 2008.

[4] I. Y. Park, B. H. Sohn, E. Yu et al., "Aberrant epigenetic modifications in hepatocarcinogenesis induced by hepatitis B virus X protein," Gastroenterology, vol. 132, no. 4, pp. 14761494, 2007.

[5] J. S. Lim, S. H. Park, and K. L. Jang, "Hepatitis C virus core protein overcomes stress-induced premature senescence by down-regulating p16 expression via DNA methylation," Cancer Letters, vol. 321, no. 2, pp. 154-161, 2012.

[6] R. Nagashio, E. Aral, H. Ojima, T. Kosuge, Y. Kondo, and Y. Kanai, "Carcinogenic risk estimation based on quantification of DNA methylation levels in liver tissue at the precancerous stage," International Journal of Cancer, vol. 129, no. 5, pp. 1170-1179, 2011.

[7] S. A. Leon, B. Shapiro, D. M. Sklaroff, and M. J. Yaros, "Free DNA in the serum of cancer patients and the effect of therapy," Cancer Research, vol. 37, no. 3, pp. 646-650, 1977.

[8] D. E. Matei and K. P. Nephew, "Epigenetic therapies for chemoresensitization of epithelial ovarian cancer," Gynecologic Oncology, vol. 116, no. 2, pp. 195-201, 2010.

[9] V. V. Levenson and A. A. Melnikov, "DNA methylation as clinically useful biomarkers - light at the end of the tunnel," Pharmaceuticals, vol. 5, no. 1, pp. 94-113, 2012.

[10] K. Jung, M. Fleischhacker, and A. Rabien, "Cell-free DNA in the blood as a solid tumor biomarker: a critical appraisal of the literature," Clinica Chimica Acta, vol. 411, no. 21-22, pp. 1611-1624, 2010.

[11] E. Dulaimi, J. Hillinck, I. Ibanez de Caceres, T. Al-Saleem, and P. Cairns, "Tumor suppressor gene promoter hypermethylation in serum of breast cancer patients," Clinical Cancer Research, vol. 10, no. 18, Part 1, pp. 6189-6193, 2004.

[12] F. Simmer, A. B. Brinkman, Y. Assenov et al., "Comparative genome-wide DNA methylation analysis of colorectal tumor and matched normal tissues," Epigenetics, vol. 7, no. 12, pp. 1355-1367, 2012.

[13] P. Han, Y. Fu, J. Liu et al., "Netrin-1 promotes cell migration and invasion by down-regulation of BVES expression in human hepatocellular carcinoma," American Journal of Cancer Research, vol. 5, no. 4, pp. 1396-1409, 2015.

[14] I. Guilleret, L. Losi, S. T. Chelbi et al., "DNA methylation profiling of esophageal adenocarcinoma using methylation ligation-dependent macroarray (MLM)," Biochemical and Biophysical Research Communications, vol. 479, no. 2, pp. 231$237,2016$. 
[15] C. C. Kuo, C. Y. Lin, Y. L. Shih et al., "Frequent methylation of HOXA9 gene in tumor tissues and plasma samples from human hepatocellular carcinomas," Clinical Chemistry and Laboratory Medicine, vol. 52, no. 8, pp. 1235-1245, 2014.

[16] M. Campan, D. J. Weisenberger, B. Trinh, and P. W. Laird, "MethyLight," Methods in Molecular Biology, vol. 507, pp. 325-337, 2009.

[17] M. J. Fackler, M. McVeigh, J. Mehrotra et al., "Quantitative multiplex methylation-specific PCR assay for the detection of promoter hypermethylation in multiple genes in breast cancer," Cancer Research, vol. 64, no. 13, pp. 4442-4452, 2004.

[18] N. Fujita, N. Kagara, N. Yamamoto et al., "Methylated DNA and high total DNA levels in the serum of patients with breast cancer following neoadjuvant chemotherapy are predictive of a poor prognosis," Oncology Letters, vol. 8, no. 1, pp. 397-403, 2014.

[19] S. Zopf, M. Ocker, D. Neureiter et al., "Inhibition of DNA methyltransferase activity and expression by treatment with the pan-deacetylase inhibitor panobinostat in hepatocellular carcinoma cell lines," BMC Cancer, vol. 12, p. 386, 2012.

[20] Q. Feng, S. E. Hawes, J. E. Stern et al., "DNA methylation in tumor and matched normal tissues from non-small cell lung cancer patients," Cancer Epidemiology, Biomarkers \& Prevention, vol. 17, no. 3, pp. 645-654, 2008.

[21] M. Hayashi, G. Wu, J. L. Roh et al., "Correlation of gene methylation in surgical margin imprints with locoregional recurrence in head and neck squamous cell carcinoma," Cancer, vol. 121, no. 12, pp. 1957-1965, 2015.

[22] B. Zhang, S. Liu, Z. Zhang et al., "Analysis of BRAFV600E mutation and DNA methylation improves the diagnostics of thyroid fine needle aspiration biopsies," Diagnostic Pathology, vol. 9, p. 45, 2014.

[23] C. A. Eads, K. D. Danenberg, K. Kawakami et al., "MethyLight: a high-throughput assay to measure DNA methylation," Nucleic Acids Research, vol. 28, article E32, 2000.

[24] C. A. Eads, R. V. Lord, K. Wickramasinghe et al., "Epigenetic patterns in the progression of esophageal adenocarcinoma," Cancer Research, vol. 61, no. 8, pp. 3410-3418, 2001.

[25] S. Ogino, T. Kawasaki, M. Brahmandam et al., "Precision and performance characteristics of bisulfite conversion and realtime PCR (MethyLight) for quantitative DNA methylation analysis," The Journal of Molecular Diagnostics, vol. 8, no. 2, pp. 209-217, 2006.

[26] W. B. Coleman and A. G. Rivenbark, "Quantitative DNA methylation analysis: the promise of high-throughput epigenomic diagnostic testing in human neoplastic disease," The Journal of Molecular Diagnostics, vol. 8, no. 2, pp. 152156, 2006.

[27] Z. H. Zhao, Y. C. Fan, Y. Yang, and K. Wang, "Association between Ras association domain family 1A promoter methylation and hepatocellular carcinoma: a meta-analysis," World Journal of Gastroenterology, vol. 19, no. 41, pp. 7189-7196, 2013.

[28] C. Y. Lu, S. Y. Chen, H. L. Peng, P. Y. Kan, W. C. Chang, and C. J. Yen, "Cell-free methylation markers with diagnostic and prognostic potential in hepatocellular carcinoma," Oncotarget, vol. 8, no. 4, pp. 6406-6418, 2017.

[29] Z. H. Huang, Y. Hu, D. Hua, Y. Y. Wu, M. X. Song, and Z. H. Cheng, "Quantitative analysis of multiple methylated genes in plasma for the diagnosis and prognosis of hepatocellular carcinoma," Experimental and Molecular Pathology, vol. 91, no. 3, pp. 702-707, 2011.

[30] S. Ogino, A. T. Chan, C. S. Fuchs, and E. Giovannucci, "Molecular pathological epidemiology of colorectal neoplasia: an emerging transdisciplinary and interdisciplinary field," Gut, vol. 60, no. 3, pp. 397-411, 2011.

[31] X. Y. Huang, J. G. Yao, B. C. Huang, Y. Ma, Q. Xia, and X. D. Long, "Polymorphisms of a disintegrin and metalloproteinase with thrombospondin motifs 5 and aflatoxin B1-related hepatocellular carcinoma," Cancer Epidemiology, Biomarkers \& Prevention, vol. 25, no. 2, pp. 334-343, 2016.

[32] S. Ogino, R. Nishihara, T. J. VanderWeele et al., "The role of molecular pathological epidemiology in the study of neoplastic and non-neoplastic disease in the era of precision medicine," Epidemiology, vol. 27, no. 4, pp. 602-611, 2016. 


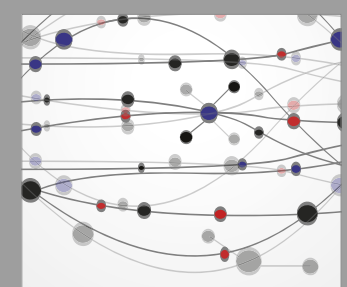

The Scientific World Journal
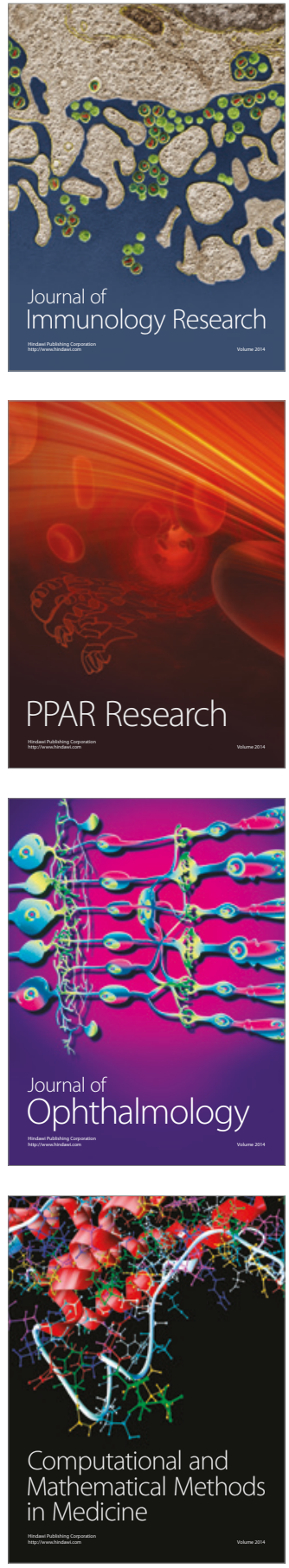

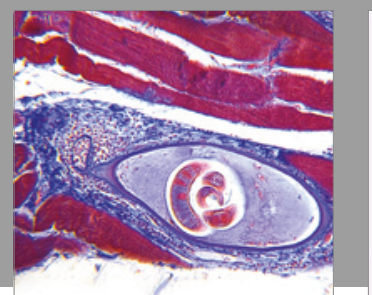

Gastroenterology Research and Practice
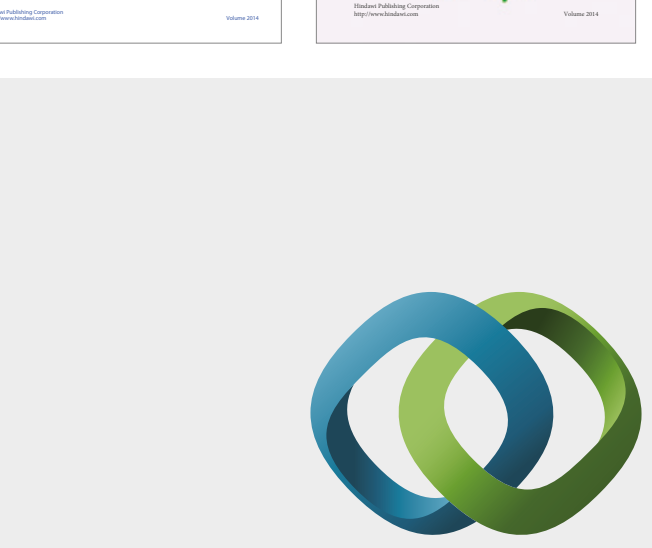

\section{Hindawi}

Submit your manuscripts at

https://www.hindawi.com
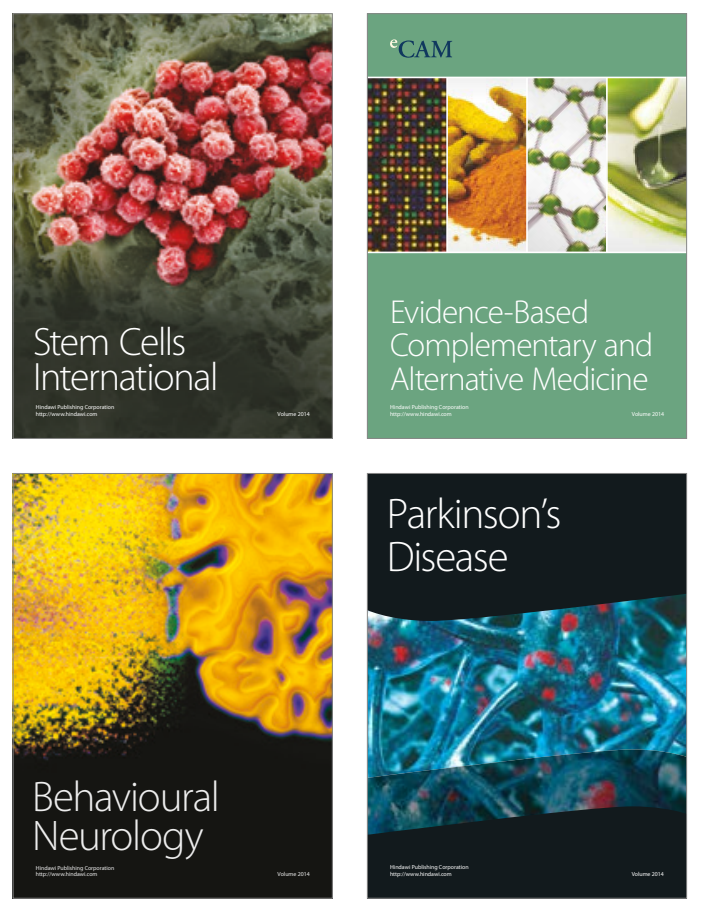
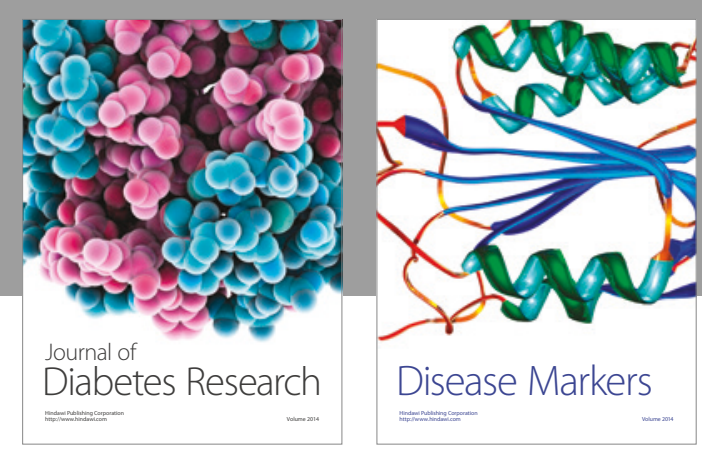

Disease Markers
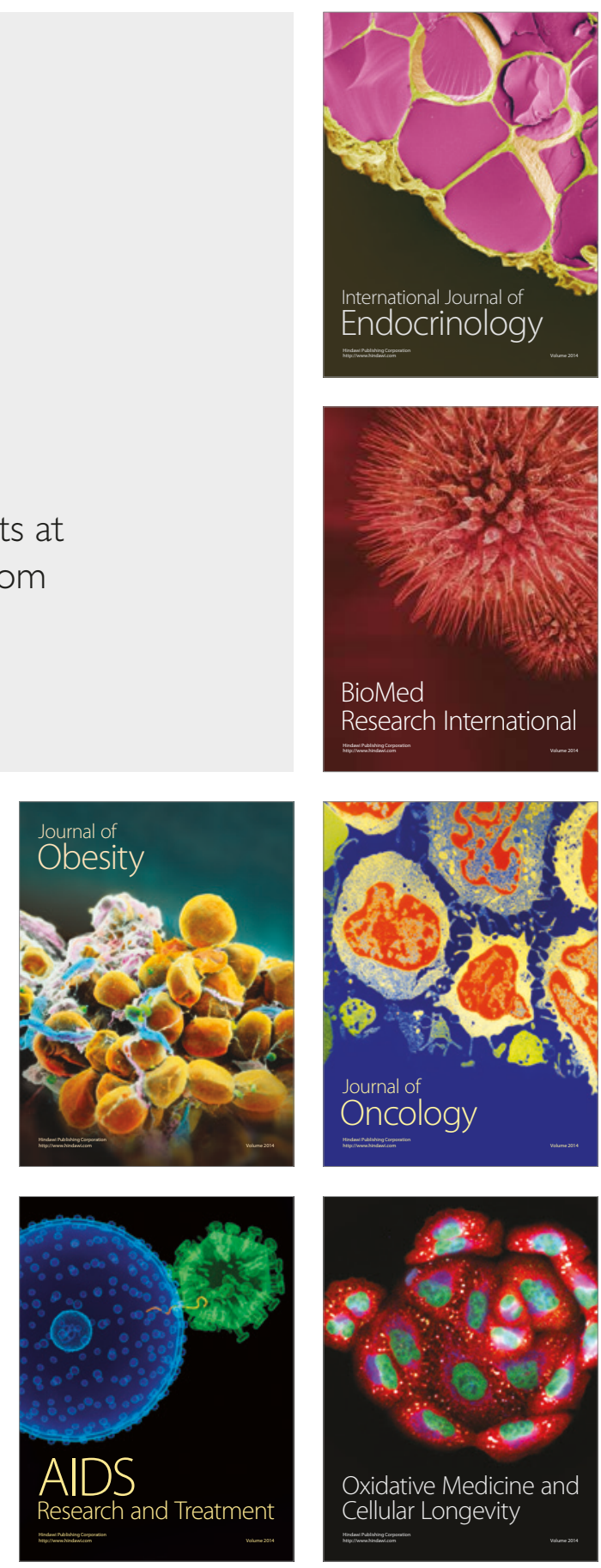